\title{
Andere spezifische Diabetesformen
}

\author{
Greisa Vila · Alois W. GessI · Michaela Riedl · Anton Luger
}

Online publiziert: 7. April 2016

(C) The Author(s) 2016. This article is available at SpringerLink with Open Access

\begin{abstract}
Zusammenfassung Zahlreiche endokrine Erkrankungen können Störungen des Kohlenhydratstoffwechsels bewirken und zur Manifestation eines Diabetes mellitus führen bzw. diese begünstigen. Mit Ausnahme der Hyperthyreose, bei der dies nur in Ausnahmefällen $\mathrm{zu}$ beobachten ist, sind diese Erkrankungen selten. Akromegalie und Cushing-Syndrom sind besonders häufig mit gestörter Glukosetoleranz oder Diabetes assoziiert, bei Phäochromozytom und Conn-Syndrom stellt dies die Ausnahme dar. Bei Medikamenten, die zur Manifestation eines Diabetes führen können, sind in erster Linie Hormone, atypische Antipsychotika und Immunsuppressiva anzuführen. Weiters kommen Pankreaserkrankungen wie Pankreatitis, Pankreaskarzinom, zystische Fibrose und Hämochromatose als sekundäre Diabetesursachen in Frage, ebenso wie das Down-Syndrom, das Klinefelter-Syndrom, das Turner-Syndrom und das Prader-Willi-Syndrom sowie andere seltenere immunmediierte oder genetische Syndrome.
\end{abstract}

Schlüsselwörter Sekundärer Diabetes mellitus · Akromegalie $\cdot$ Cushing-Syndrom · medikamenteninduzierter Diabetes mellitus

\section{Other specific types of diabetes}

Summary Numerous endocrine diseases are associated with impaired glucose metabolism and can induce diabetes mellitus. With the exception of hy-

G. Vila · A. W. Gessl · M. Riedl · A. Luger $(\bowtie)$

Klinische Abteilung für Endokrinologie und Stoffwechsel,

Universitätsklinik für Innere Medizin III, Medizinische

Universität und Allgemeines Krankenhaus Wien

Wien, Österreich

E-Mail: anton.luger@meduniwien.ac.at perthyroidism, where this is uncommon, these diseases are rare. Acromegaly and Cushing syndrome are frequently associated with impaired glucose tolerance and diabetes. In contrast, this is a rare finding in pheochromocytoma and Conn syndrome. Among the many drugs that can induce diabetes this can be observed most frequently with hormones, atypic antipsychotic drugs and immunosuppressives. In addition, diseases of the pancreas such as pancreatitis, pancreatic carcinoma, cystic fibrosis and hemochromatosis can cause diabetes as well as Down syndrome, Klinefelter syndrome, Turner syndrome and Prader Willi syndrome and rare immunmediated or genetic syndromes.

Keywords Secondary diabetes mellitus · Acromegaly · Cushing-syndrome · Drug-induced diabetes mellitus

Neben den an anderer Stelle behandelten genetischen Defekten der Beta-Zell-Funktion und Insulin-Wirkung sollen in diesem Beitrag endokrine Erkrankungen und Medikamente, die Diabetes verursachen können, behandelt werden. Weiters auch Pankreas-Erkrankungen, die nicht primär durch einen Defekt der BetaZellen verursacht sind, und schließlich sollen noch seltene andere Ursachen kurz erwähnt werden, wie Infektionen, Autoimmunerkrankungen (ausgenommen Diabetes mellitus Typ 1) und genetische Syndrome, die mit Diabetes assoziiert sein können.

\section{Endokrine Erkrankungen}

Von den Endokrinopathien, die einen sekundären Diabetes verursachen können, sind in erster Linie die Akromegalie und das Cushing-Syndrom zu erwähnen [1-3]. Weitere hormonelle Erkrankungen, wie 
Tab. 1 Labor-Diagnostik zum Ausschluss von Endokrinopathien

\begin{tabular}{|c|c|}
\hline \multirow[t]{2}{*}{ Akromegalie } & IGF-1 \\
\hline & GH bei Glucose-Suppressionstest \\
\hline \multirow[t]{5}{*}{ Cushing-Syndrom } & Suchtests: \\
\hline & Cortisol im 24 h-Harn \\
\hline & 1 mg Dexamethason-Hemmtest \\
\hline & Serum/Speichel-Cortisol um 23.00/24.00 Uhr \\
\hline & $\begin{array}{l}\text { Danach Spezialtests zur Differentialdiagnose hypo- } \\
\text { physäre, adrenale oder andere Ursache }\end{array}$ \\
\hline \multirow[t]{2}{*}{ Phäochromozytom } & Metanephrine/Normetanephrine im Harn/Plasma \\
\hline & Clonidin-Test \\
\hline Morbus Conn & Aldosteron im Serum, Renin im Plasma \\
\hline Hyperthyreose & $\mathrm{TSH}, \mathrm{fT}_{4}, \mathrm{~T}_{3}$ \\
\hline Glukagonom & Glukagon-Plasmakonzentration \\
\hline Somatostatinom & Somatostatin-Plasmakonzentration \\
\hline
\end{tabular}

Tab. 2 Klinische Symptome der Akromegalie (Metaanalyse, 569 Patienten) [4]

\begin{tabular}{|l|l|}
\hline Akrales Wachstum & $96-100 \%$ \\
\hline Hyperhydrose & $60-88 \%$ \\
\hline Cephalea & $50-87 \%$ \\
\hline Parästhesien & $30-79 \%$ \\
\hline Diabetes mellitus, gestörte Glukosetoleranz & $25-60 \%$ \\
\hline Kardiovaskuläre Erkrankungen & $12-34 \%$ \\
\hline Hypertonie & $18-32 \%$ \\
\hline Struma & $18-41 \%$ \\
\hline Dysmenorrhoe & $32-87 \%$ \\
\hline Libido-, Potenzstörungen & $27-46 \%$ \\
\hline Gesichtsfeldeinschränkungen & $6-62 \%$ \\
\hline
\end{tabular}

das Phäochromozytom, das Conn-Syndrom und die Hyperthyreose haben einen vergleichsweise geringen Einfluss auf den Kohlenhydratstoffwechsel, das Glukagonom und Somatostatinom sind so selten, dass sie nur kurz Erwähnung finden sollen. Gemeinsam ist diesen Erkrankungen, dass die Behandlung der zugrundeliegenden Erkrankung häufig zur Besserung oder Heilung der diabetischen Stoffwechselentgleisung führt. Wenngleich somit Diagnostik (Tab. 1) und Therapie der auslösenden Endokrinopathie im Vordergrund stehen, darf die Therapie des Diabetes nicht vernachlässigt werden.

\section{Akromegalie}

Eine überschießende Produktion von Somatotropin ist zumeist auf ein Somatotropin (GH)-produzierendes Hypophysen-Adenom zurückzuführen, sehr selten auch auf ektope Produktion des hypothalamischen Releasing-Hormons für Somatotropin-GHRH.

Die somatischen Veränderungen bilden sich nur langsam aus, sodass die Diagnose im Durchschnitt erst 7-10 Jahre nach den retrospektiv eruierbaren ersten Symptomen gestellt wird. Die zahlreichen mögli- chen klinischen Symptome der Akromegalie sind in Tab. 2 zusammengestellt [4].

Aufgrund der pulsatilen Freisetzung von GH ist bei nicht eindeutiger Befundkonstellation (klinische Symptome und extrem erhöhte GH- und IGF-I-Werte) ein GH-Suppressionstest erforderlich (ident mit oralem Glukosetoleranz-Test: 75 mg Glukose peroral) mit Bestimmung von $\mathrm{GH}$ vor und alle $30 \mathrm{~min}$ bis $2 \mathrm{~h}$ nach Glukoseingestion. Die Diagnose kann bei Suppression der GH-Konzentration unter $1 \mathrm{ng} / \mathrm{ml}$ ausgeschlossen werden. Aufgrund der längeren Halbwertszeit ist die unter Einfluss von GH stehende IGF-I-Konzentration zwar besser zur Diagnose verwertbar, jedoch unterliegt auch diese zahlreichen Einflüssen (u. a. Ernährung, Begleiterkrankungen).

\section{Cushing-Syndrom}

Ein Hypercortisolismus ist in der überwiegenden Mehrzahl der Fälle auf eine vermehrte ACTH-Produktion zurückzuführen, die wiederum zumeist durch ein ACTH-produzierendes Hypophysen-Adenom bedingt ist. In $20 \%$ der Fälle ist jedoch eine ektope Quelle die Ursache (meist kleinzelliges BronchusKarzinom). Adrenale Ursache für einen Hypercortisolismus ist meist ein Nebennieren-Adenom, seltener ein Karzinom oder eine mikro- oder makronoduläre Hyperplasie. Die zahlreichen klinischen Zeichen und Symptome sind in Tab. 3 zusammengefasst.

Die Differentialdiagnose des Cushing-Syndroms stellt häufig eine Herausforderung dar und sollte im Gegensatz zur Basisdiagnostik (1 mg DexamethasonHemmtest, Cortisol-Ausscheidung im 24 h-Harn, Cortisol-Konzentration im Serum oder Speichel um 23.00 oder 24.00 Uhr) in spezialisierten Zentren erfolgen. Zur weiteren differentialdiagnostischen Abklärung sind der CRH-Test (Verabreichung des hypothalamischen Releasing-Hormons für ACTH - CorticotropinReleasing-Hormon [CRH] und Messung von ACTH und Cortisol alle halben Stunden über 2 h) bzw. der Liddle-Test geeignet. Bei Letzterem werden über 2 Ta-

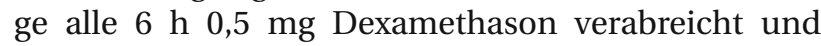
daran anschließend über 2 Tage alle 6 h 2 mg Dexamethason. Cortisol ist bei einem auf ein Hypophysen-Adenom zurückzuführenden Hypercortisolismus supprimierbar und ACTH im CRH-Test gesteigert stimulierbar, wohingegen ACTH bei adrenal verursachtem Cushing-Syndrom durch $\mathrm{CRH}$ nicht stimulierbar und Cortisol im Liddle-Test nicht supprimierbar ist. Beim ektopen Cushing-Syndrom ist eine basal hohe bis sehr hohe ACTH-Konzentration durch CRH nicht stimulierbar und Cortisol im Liddle-Test nicht supprimierbar.

\section{Phäochromozytom}

Meist ist die Beeinträchtigung des Kohlenhydratstoffwechsels durch vermehrte Produktion von Adrenalin 
leitlinien für die praxis

Tab. 3 Cushing-Syndrom-Symptome

\begin{tabular}{ll}
\hline Allgemein & \multicolumn{1}{l}{ Stammfettsucht } \\
\hline Vollmondgesicht & Hypertonie \\
\hline Büffelnacken & Kopfschmerzen \\
\hline Geschwächte Immunabwehr & Ulcus ventriculi \\
\hline Haut & Hautatrophie \\
\hline Plethora \\
\hline Hirsutismus \\
\hline Striae \\
\hline Suffusionen \\
\hline Regelanomalie \\
\hline Impotenz bzw. \\
\hline Verminderte Libido \\
\hline Osteopenie - Osteoporose \\
\hline Muskel \& Skelett & Muskelschwäche \\
\hline Metabolisch & Gestörte Glukosetoleranz/Diabetes mellitus \\
\hline Hyperlipidämie \\
\hline Depression \\
\hline Psychosen \\
\hline
\end{tabular}

Tab. 4 Medikamente, die zur Manifestation eines Diabetes führen können

\begin{tabular}{|l|l|}
\hline Hormone & Glucocorticoide \\
\hline & T3 und T4 \\
\hline Somatotropin \\
\hline Megestrolacetat \\
\hline Atypische Antipsychotika & Somatostatin-Analoga \\
\hline Olanzapin \\
\hline Clozapin \\
\hline Immunsuppressiva & Risperidon \\
\hline Quetiapin \\
\hline Antikonvulsiva & Cyclosporin \\
\hline Proteaseinhibitoren & Tacrolimus \\
\hline Statine & Sirolimus \\
\hline Nikotinsäure & Interferon gamma \\
\hline Diuretika & Phenytoin \\
\hline Antihypertensiva & Petablocker \\
\hline & Clonidin \\
\hline & L-Asparaginase \\
\hline & Thiazide \\
\hline
\end{tabular}

und/oder Noradrenalin nicht stark ausgeprägt, selten kommt es zur Manifestation eines Diabetes.

Die Diagnose sollte durch Nachweis von erhöhten Metanephrin- und Normetanephrin-Konzentrationen im Plasma (alternativ im 24 h-Harn) erfolgen. Bei Verwendung von Plasmametanephrin ist heute nur mehr sehr selten bei nicht eindeutiger Konstellation ein Clonidin-Hemmtest erforderlich.

\section{Conn-Syndrom}

Auch beim Conn-Syndrom ist die Beeinträchtigung des Kohlenhydratstoffwechsels meist nicht stark ausgeprägt. Die Diagnose eines primären Aldosteronismus ist aber ungleich schwieriger als jene des Phäochromozytoms. Als Suchtest sollten primär die ReninPlasma-Konzentration und die Serum-AldosteronKonzentration bestimmt werden [5]. Für das ConnSyndrom ist ein erhöhter Aldosteron-Renin-Quotient charakteristisch, Grenzwerte sind für einzelne Labors unterschiedlich, Renin ist meist unterdrückt. Eine Herausforderung stellt hier insbesondere die gleichzeitige Notwendigkeit der Behandlung einer häufig stark ausgeprägten Hypertonie und die Interferenz nahezu aller Antihypertensiva mit dem Renin-Angiotensin-Aldosteron-System dar. Jedenfalls abzusetzen ist Spironolacton (und Eplerenon), da diese in den meisten gegenwärtig verfügbaren Bestimmungsmethoden mit Aldosteron kreuzreagieren. Aufgrund der Halbwertszeit von Spironolacton ist eine Latenz von etwa 4 Wochen zu berücksichtigen. Bei nicht eindeutiger Befundkonstellation ist ein Salz-Belastungstest durchzuführen, der wahlweise durch Kochsalz-Infusion oder orale Verabreichung von Kochsalz erfolgen kann [5].

\section{Hyperthyreose}

Auch die Hyperthyreose ist selten mit ausgeprägten Störungen des Kohlenhydratstoffwechsels vergesellschaftet, jedoch kann ein vorbestehender Diabetes aggraviert bzw. auch eine Manifestation ausgelöst werden. Die Diagnose der Hyperthyreose erfolgt standardgemäß mit Bestimmung von TSH sowie von freiem $\mathrm{T} 4$ und $\mathrm{T} 3$.

\section{Glukagonom und Somatostatinom}

Beide Erkrankungen stellen extrem seltene Tumore des Pankreas dar, ausgehend von Alpha-Zellen bzw. Delta-Zellen. Die Auswirkungen auf den Kohlenhydratstoffwechsel können sehr ausgeprägt sein. Zur Diagnose des Glukagonoms führen zumeist Hautveränderungen (nekrolytisch, migratorisches Erythem), das vorwiegend perioral, perianal bzw. perigenital auftritt und mit Zink-Mangel verwechselt werden kann. Die Tumore sind bei Diagnose meistens bereits sehr groß, die Diagnostik erfolgt durch Nachweis von Glukagon bzw. Somatostatin im Plasma. 
Tab. 5 Pankreaserkrankungen, die zur Manifestation eines Diabetes führen können

\begin{tabular}{l} 
Pankreatitis \\
\hline Pankreas-Karzinom \\
\hline Zystische Fibrose \\
\hline Hämochromatose \\
\hline Fibrokalkuläre Pankreatopathie
\end{tabular}

Tab. 6 Immunmediierte und genetische Syndrome, die mit Diabetes assoziiert sein können [1,2]

\begin{tabular}{l} 
1) „Stiff-man“-Syndrom \\
2) Anti-Insulin-Rezeptor-Antikörper \\
3) Down-Syndrom \\
\hline 4) Klinefelter-Syndrom \\
5) Turner-Syndrom \\
6) Wolfram-Syndrom \\
\hline 7) Friedreich-Ataxie \\
8) Huntington-Chorea \\
9) Laurence-Moon-Biedl-Syndrom \\
\hline 10) Myotonie Dystrophie \\
11) Porphyrie \\
\hline 12) Prader-Willi-Syndrom \\
13) mitochondrialer Diabetes mit Taubheit (MIDD) \\
\hline
\end{tabular}

\section{Medikamente}

Die Liste jener Medikamente, die zur Manifestation eines Diabetes führen können, wird zunehmend länger ([6]; Tab. 4). Neben den aus den Endokrinopathien abzuleitenden Hormon-Präparaten sind in den letzten Jahren insbesondere die häufig mit einer sehr raschen und erheblichen Gewichtszunahme verbundenen atypischen Antipsychotika [7] zu erwähnen, aber auch die nach Organ-Transplantation zum Einsatz kommenden Immunsuppressiva [8]. Auch Statine wurden als Ursache für das vermehrte Auftreten von Diabetes identifiziert, das Risiko der Diabetes-Manifestation wird jedoch durch die benefiziellen Wirkungen der Statine bei Weitem überwogen [9]. Diuretika und weitere in Tab. 4 angeführte Pharmaka werden ebenfalls mit einer gehäuften Diabetes-Inzidenz in Zusammenhang gebracht, jedoch ist ihr Effekt meist gering bis vernachlässigbar [10].

\section{Erkrankungen des exokrinen Pankreas}

Hier ist in erster Linie die Pankreatitis jedweder Ursache als auslösender Faktor zu erwähnen ([1, 2]; Tab. 5). Ebenso können die an Inzidenz zunehmenden Pankreas-Karzinome auch bei geringer Tumorlast und somit fehlender räumlicher Beeinträchtigung von BetaZellen mit einer ausgeprägten Störung des Kohlenhydratstoffwechsels verbunden sein.

Aufgrund der erfolgreichen Behandlung von Patienten mit zystischer Fibrose und der damit verbundenen höheren Lebenserwartung, häufig in Verbindung mit Lungen-Transplantation und dadurch erforderli- cher Immunsuppression, nimmt auch diese Gruppe von Patienten mit sekundärem Diabetes rasch zu [11].

Als weitere mögliche Ursachen sind hier noch die Hämochromatose und die fibrokalkuläre Pankreatopathie zu erwähnen [1, 2]. Letztere wird in Österreich jedoch kaum beobachtet werden können.

\section{Infektionen und seltene andere Ursachen}

Eine Reihe von viralen Erkrankungen kann mit Zerstörung der Beta-Zellen einhergehen. Zu erwähnen sind hier Röteln, Coxsackie-Virus B, Zytomegalie-Virus, Adeno-Virus und Mumps. Seltene immunmediierte Diabetes-Formen sowie im Rahmen von seltenen genetischen Syndromen auftretende sind in Tab. 6 angeführt $[1,2]$.

\section{Einhaltung ethischer Richtlinien}

\section{Interessenkonflikt}

G. Vila, A. W. Gessl, M. Riedl und A. Luger geben an, dass kein Interessenkonflikt besteht.

\section{Open Access}

Dieser Artikel unterliegt den Bedingungen der Creative Commons Attribution License. Dadurch sind die Nutzung, Verteilung und Reproduktion erlaubt, sofern der/die Originalautor/en und die Quelle angegeben sind.

\section{Literatur}

1. American Diabetes Association. Diagnosis and classification of Diabetes mellitus. Diabetes Care. 2013;36(Suppl 1):S67-S74.

2. American Diabetes Association. 2. Classification and Diagnosis of Diabetes. Diabetes Care. 2015;38(Suppl 1):S8-S16.

3. Resmini E, Minuto F, Colao A, et al. Secondary diabetes associated with principal endocrinopathies: the impact of new treatment modalities. Acta Diabetol. 2009;46:85-95.

4. Jadresic A, Banks LM, Child DF, et al. The acromegaly syndrome. Relation between clinical features, growth hormone values and radiological characteristics of the pituitary tumours. QJ Med. 1982;51:189-204 (Spring).

5. Schirpenbach C, Reincke M. Screening for primary aldosteronism. Best Pract Res Clin Endocrinol Metab. 2006;20:369-84.

6. McCulloch DK, Robertson RP. Pathogenesis of type 2 Diabetes. UpToDate. 2015;64:1261.

7. Al-Zoairy R, Ress C, Tschoner A, et al. The effects of psychotropic drugs on the regulation of glucose metabolism. Curr Diabetes Rev. 2013;9:362-70.

8. Jenssen T, Hartmann A. Emerging treatments for posttransplantation diabetes mellitus. Nat Rev Nephrol. doi:10.1038/nrneph.2015.59.

9. Chogtu B, Magazine R, Bairy KL. Statin use and risk of diabetes mellitus. World J Diabetes. 2015;6:352-75.

10. Singh P, Knoedler JJ, Krambeck AE, et al. Thiazide diuretic prophylaxis for kidney stones and the risk of diabetes mellitus. J Urol. 2014;192:1700-4.

11. Moran A, Brunzell C, Cohen RC, et al. Clinical Care Guidelines for Cystic Fibrosis-Related Diabetes. Diabetes Care. 2010;33(12):2697-708. 